OPEN ACCESS

Edited by:

Dongfeng Zhang,

Qingdao University, China

Reviewed by:

Yaofei Sun,

Weifang People's Hospital, China

Changwei Tian,

Kunshan Municipal Centers for

Disease Control and Prevention, China

*Correspondence:

Jun Shao

baitiantougua123@126.com

Specialty section:

This article was submitted to

Nutritional Epidemiology,

a section of the journal

Frontiers in Nutrition

Received: 19 August 2021

Accepted: 25 October 2021

Published: 11 November 2021

Citation:

Liu X, Shen H, Chen M and Shao J (2021) Clinical Relevance of Vitamins and Carotenoids With Liver Steatosis

and Fibrosis Detected by Transient Elastography in Adults.

Front. Nutr. 8:760985.

doi: 10.3389/fnut.2021.760985

\section{Clinical Relevance of Vitamins and Carotenoids With Liver Steatosis and Fibrosis Detected by Transient Elastography in Adults}

\author{
Xiaohui Liu, Hong Shen, Mingfeng Chen and Jun Shao* \\ Department of Ultrasound Diagnosis, The First People's Hospital of Kunshan, Kunshan Hospital Affiliated to Jiangsu \\ University, Kunshan, China
}

Background: Vitamins and carotenoids may be involved in the pathogenesis of non-alcoholic fatty liver disease (NAFLD). Previously related publications mainly focused on vitamin $\mathrm{D}$ and vitamin $\mathrm{E}$, and studies on other vitamins and carotenoids and NAFLD are scarce.

Methods: This study aimed to explore the clinical relevance of vitamin A, B vitamins (vitamin B1, vitamin B2, niacin, vitamin B6, folate, vitamin B12, and choline), vitamin C and carotenoids ( $\alpha$-carotene, $\beta$-carotene, $\beta$-cryptoxanthin, lycopene, lutein + zeaxanthin) with liver steatosis and fibrosis in the 2017-2018 NHANES ( $N=4,352)$. Liver steatosis and fibrosis were detected by transient elastography. Logistic regression, linear regression and restricted cubic splines were adopted to explore the non-linear dose-response relationships.

Results: Higher intakes of vitamin C [0.68 (0.50-0.93)] and $\beta$-carotene [0.71 (0.54-0.93)] were inversely associated with liver steatosis. Higher levels of serum vitamin C [0.45 (0.32-0.62)] were inversely associated with liver fibrosis, while higher intakes of choline [1.43 (1.04-1.98)] and $\alpha$-carotene [1.67 (1.01-2.74)] were positively associated with liver fibrosis. In addition, marginally inverse association between lutein + zeaxanthin and liver steatosis and positive association between vitamin B12 and liver fibrosis were found. In linear regression, the above-mentioned associations between vitamin $\mathrm{C}, \beta$-carotene, and lutein + zeaxanthin and liver steatosis, and serum vitamin $C$, choline, $\alpha$-carotene, and vitamin B12 and liver fibrosis were also found. The above-mentioned associations were mainly linear, while the relationship between $\beta$-carotene and liver steatosis might be non-linear.

Conclusion: Vitamin C, $\alpha$-carotene, $\beta$-carotene, lutein + zeaxanthin, choline and vitamin B12 may be associated with liver steatosis and fibrosis.

Keywords: carotenoids, liver fibrosis, liver steatosis, dose-response analysis, vitamins, national health and nutrition examination survey 


\section{INTRODUCTION}

Non-alcoholic fatty liver disease (NAFLD) is a major cause of liver disease worldwide, and $25 \%$ of the adult population in the world has NAFLD (1). NAFLD ranges from simple steatosis to non-alcoholic steatohepatitis with or without hepatic fibrosis in the absence of excessive alcohol intake (2). Although substantial advances have been made in NAFLD mechanisms, diagnostics, and treatment (2), NAFLD remains the center of attention within liver owing to its high prevalence and growing burden in the general population (3). The most effective type of longterm treatment of NAFLD remains lifestyle modification $(4,5)$. Although the pathogenesis of NAFLD is not well-understood, the leading hypothesis is the hepatic fat accumulation owing to insulin resistance followed by oxidative stress arising from reactive oxygen species produced primarily by mitochondria $(6,7)$. The liver plays a critical role in the metabolism of vitamins, and deregulation of vitamins metabolism may contribute to the initiation and progression of NAFLD (6-8). The evidence available supports the potential benefits of vitamin D and vitamin E on NAFLD development $(9,10)$. However, studies on other vitamins and carotenoids and NAFLD are scarce. Transient elastography is considered the non-invasive standard tool for assessing liver fibrosis (11), and provides higher sensitivity and specificity for detecting liver steatosis than liver enzymes (12), and has been adopted to detect liver steatosis and liver fibrosis in the general population $(13,14)$. Therefore, based on the 2017-2018 National Health and Nutrition Examination Survey (NHANES), we aimed to examine the clinical relevance of vitamin $\mathrm{A}, \mathrm{B}$ vitamins (vitamin B1, vitamin B2, niacin, vitamin B6, folate, vitamin B12, and choline), vitamin C and carotenoids $(\alpha$-carotene, $\beta$-carotene, $\beta$-cryptoxanthin, lycopene, lutein + zeaxanthin) with liver steatosis and fibrosis detected by transient elastography in adults.

\section{MATERIALS AND METHODS}

\section{Eligible Sample}

NHANES is a national population-based survey program assessing the health and nutritional status of the civilian noninstitutionalized general U.S. population. The NHANES data are released every 2 years. Data from the 2017-2018 NHANES cycle were used in this analysis, because this cycle specifically provided data for liver steatosis and fibrosis.

All participants aged 20 years and over were eligible. Participants were excluded if (1) their dietary intakes of vitamins, carotenoids, or serum vitamin $\mathrm{C}$ levels are missing; (2) they are infected with hepatitis B or C (defined by the presence of hepatitis $\mathrm{C}$ antibodies or the presence of hepatitis B surface antigen); (3) they have significant alcohol consumption ( $>30 \mathrm{~g} /$ day in men and $>20 \mathrm{~g} /$ day in women) (13); and (4) their data of transient elastography are missing. The protocol was approved by the National Center for Health Statistics Institutional Review Board, and all subjects provided written informed consent.

\section{Vitamins and Carotenoids}

Dietary intakes of vitamin A, B vitamins (vitamin B1, vitamin $\mathrm{B} 2$, niacin, vitamin $\mathrm{B} 6$, folate, vitamin $\mathrm{B} 12$, and choline), vitamin $\mathrm{C}$ and carotenoids ( $\alpha$-carotene, $\beta$-carotene, $\beta$ cryptoxanthin, lycopene, lutein + zeaxanthin) were included as exposures. All NHANES participants are eligible for two 24$\mathrm{h}$ dietary recall interviews. The first dietary recall interview is collected in-person in the Mobile Examination Center and the second interview is collected by telephone 3-10 days later. In this analysis, dietary intakes of vitamin and carotenoids were assessed using 1-day values for individuals with single recalls and 2-day means for others.

Serum vitamin $\mathrm{C}$ concentrations are also available in the dataset, and thus were also included in this analysis. Vitamin $\mathrm{C}$ in serum is measured using isocratic ultra-high performance liquid chromatography. Serum specimens were processed, stored, and shipped to the Division of Laboratory Sciences, National Center for Environmental Health, Centers for Disease Control and Prevention, Atlanta, GA for analysis. Vials are stored under appropriate frozen $\left(-30^{\circ} \mathrm{C}\right)$ conditions until they are shipped to National Center for Environmental Health for testing. The NHANES quality assurance and quality control protocols meet the 1988 Clinical Laboratory Improvement Act mandates.

\section{Transient Elastography}

The main goals of the transient elastography component are to provide objective measures for two important liver disease manifestations: liver fibrosis and liver steatosis. Liver fibrosis was measured by FibroScan ${ }^{\circledR}$ which uses ultrasound and the vibration controlled transient elastography to derive liver stiffness. The device also simultaneously measures the ultrasound attenuation related to the presence of liver steatosis and records the controlled attenuation parameter as the indicator for the fatness in the liver. The elastography exam was performed by NHANES health technicians, who were trained and certified by NHANES staff and the equipment manufacturer. A detailed description of quality assurance and quality control measures considered for this component can be found in the Procedures Manual (15). We defined liver steatosis as controlled attenuation parameter scores of $263 \mathrm{~dB} / \mathrm{m}$ or more and liver stiffness values of $8 \mathrm{kPa}$ or higher were considered to have significant fibrosis $(11,16,17)$.

\section{Covariates}

According to the previously related publication (13), we included the following covariates that are risk factors of NAFLD: age (in 10-year increments), sex, race/ethnicity (Mexican-American, Other Hispanic, Non-hispanic White, Non-hispanic Black, Other Races), education ( $\leq$ high school, $>$ high school), recreational physical activity (at least $10 \mathrm{~min}$ continuously per week), smoking (current smoker, former smoker, never smoker), alcohol drinking (continuous), hypertension, diabetes, body mass index (under/normal weight: $<25 \mathrm{~kg} / \mathrm{m}^{2}$, overweight: $25-<30 \mathrm{~kg} / \mathrm{m}^{2}$, obesity: $\geq 30 \mathrm{~kg} / \mathrm{m}^{2}$ ), and dietary intakes of cholesterol. Diabetes was defined using a previous diagnosis of diabetes or taking diabetic pills to lower blood sugar or, if diabetes was not previously diagnosed, by a hemoglobin $\mathrm{A} 1 \mathrm{c}$ level $\geq 6.5 \%$, or a fasting plasma glucose level $\geq 126 \mathrm{mg} / \mathrm{dL}$ (18). Subjects were considered hypertensive if they were taking antihypertensive medication, if their systolic blood pressure exceeded $130 \mathrm{mmHg}$, 
or if their mean diastolic blood pressure exceeded $80 \mathrm{mmHg}$ (mean values of three measurements) (19).

\section{Statistical Analysis}

Logistic regression was adopted to explore the associations between vitamins and carotenoids and liver steatosis and fibrosis. Subjects were classified into tertiles according to their dietary intakes of vitamins, carotenoids or serum vitamin $\mathrm{C}$ concentrations, and the odds ratios (ORs) and 95\% confidence intervals (CIs) of liver steatosis and fibrosis for subjects in tertile 2 and tertile 3 were calculated as compared to those in tertile 1 . There are three different logistic regression models. Model 1 was adjusted for age, sex and race/ethnicity. Model 2 was adjusted for covariates in model 1 , and also education, physical activity, smoking and alcohol drinking. Model 3 was adjusted for covariates in model 2, and also body mass index, hypertension, diabetes, and dietary intakes of cholesterol. The potential non-linear dose-response relationships between vitamins, carotenoids, and liver steatosis and fibrosis were examined by modeling dietary intakes of vitamins, carotenoids, or serum vitamin $\mathrm{C}$ concentrations using restricted cubic splines with 3 knots at percentiles 25, 50, and 75 of the distribution (20). A $P$-value for non-linearity was calculated by testing the null hypothesis that the coefficient of the second spline is equal to 0 (20). We also conducted multivariable linear regression model with the outcomes and exposures expressed as continuous variables. In sensitivity analysis, we explored the associations between vitamin intakes from both diets and supplements and liver steatosis and liver fibrosis. All analyses were conducted using STATA version 12.0 , and $P \leq 0.05$ was considered statistically significant. Appropriate strata, cluster, and weights were considered in all analyses as suggested by NHANES.

\section{RESULTS}

\section{Population Characteristics}

According to the exclusion criteria, individuals who are infected with hepatitis $\mathrm{B}$ or $\mathrm{C}(N=79)$, who they have significant alcohol consumption $(N=457)$ and whose data of transient elastography are missing $(N=630)$, a total of 4,352 subjects were potentially eligible for this analysis. Among the 4,352 subjects, 4,116 provided data of serum vitamin $C$ concentrations and 3,940 provided dietary intakes of vitamins and carotenoids. The weighted prevalence of was $56.47 \%$ for liver steatosis and $15.05 \%$ for liver fibrosis, respectively. Patients with liver steatosis and fibrosis showed higher prevalence of diabetes, obesity and hypertension (all $P<0.01$ ). Patients with liver fibrosis and liver steatosis had higher dietary intakes of vitamin B12, choline and cholesterol, while had lower dietary intakes of vitamin $\mathrm{C}$ and lutein + zeaxanthin and lower levels of serum vitamin $\mathrm{C}$ concentrations (all $P \leq 0.05$; Table 1 ).

\section{Associations With Liver Steatosis}

Overall, the findings between vitamins and carotenoids and liver steatosis were similar between model 1 and model 2 . In model 2, comparing the highest to lowest tertile, higher dietary intakes of vitamin C [OR ( $95 \%$ CI): $0.59 \quad(0.42-$ $0.81)]$ and $\beta$-carotene $[0.70(0.57-0.87)]$ and serum vitamin $C$ concentrations $[0.50(0.38-0.66)]$ were inversely associated with liver steatosis, respectively. However, after further adjustment for obesity, diabetes, hypertension and cholesterol intakes (model $3)$, only dietary intakes of vitamin $C[0.68(0.50-0.93)]$ and $\beta$ carotene $[0.71(0.54-0.93)]$ were significantly associated with liver steatosis. In addition, a marginally inverse association between lutein + zeaxanthin and liver steatosis were found [0.74 (0.53-1.04)]. No associations were found between other vitamins and carotenoids and liver steatosis in model 3 (Table 2). The findings from multivariable linear regression model are consistent with those from the logistic regression, and the above-mentioned associations between vitamin $C, \beta$ carotene, and lutein + zeaxanthin and liver steatosis were also observed (Supplementary Table 2). Similar findings were also found in sensitivity analysis including intakes from Supplementary Table 3.

\section{Associations With Liver Fibrosis}

Overall, the findings between vitamins and carotenoids and liver fibrosis were similar between model 1 and model 2. In model 2, comparing the highest to lowest tertile, higher dietary intakes of vitamin B12 [1.49 (1.01-2.18)] and choline [1.56 (1.11-2.21)] were positively associated with liver fibrosis. In addition, higher dietary intakes of $\alpha$-carotene [tertile 2 vs. tertile 1: 1.85 (1.162.95)] were also positively associated with liver fibrosis. Higher levels of serum vitamin $\mathrm{C}$ were inversely associated with liver fibrosis [0.45 (0.29-0.70)]. After further adjustment for obesity, diabetes, hypertension and cholesterol intakes (model 3), higher dietary intakes of choline [tertile 2 vs. tertile 1: 1.43 (1.04-1.98)] were positively associated with liver fibrosis. Higher levels of serum vitamin $\mathrm{C}$ were inversely associated with liver fibrosis in model 3 [tertile 2 vs. tertile 1: $0.45(0.32-0.62)$ ].

No associations were found between other vitamins and carotenoids and liver fibrosis in model 3 (Table 3). The findings from multivariable linear regression model are consistent with those from the logistic regression, and the above-mentioned associations between serum vitamin $C$, choline, $\alpha$-carotene and vitamin B12 and liver fibrosis were also observed (Supplementary Table 2). Similar findings were also found in sensitivity analysis including intakes from Supplementary Table 4.

\section{Dose-Response Relationships}

The median values in the first category (tertile 1) of exposures were used as the references values in dose-response analysis. Dose-response analysis with restricted cubic splines showed that the departure from a linear relationship was not significant between vitamin $\mathrm{C}\left(\mathrm{P}_{\text {fornon-linearity }}=0.30\right)$ and lutein + zeaxanthin $\left(\mathrm{P}_{\text {fornon-linearity }}=0.75\right)$ and liver steatosis, while the departure from a linear relationship was significant between $\beta$-carotene $\left(\mathrm{P}_{\text {fornon-linearity }}=0.02\right)$ and liver steatosis. The departure from a linear relationship was not significant between serum vitamin $\mathrm{C}\left(\mathrm{P}_{\text {fornon-linearity }}=0.34\right)$, choline $\left(\mathrm{P}_{\text {fornon-linearity }}\right.$ $=0.36), \alpha$-carotene $\left(\mathrm{P}_{\text {fornon-linearity }}=0.36\right)$ and vitamin $\mathrm{B} 12$ (0.45) and liver fibrosis. 
TABLE 1 | Population characteristics by presence of liver steatosis and liver fibrosis.

\begin{tabular}{|c|c|c|c|c|c|c|c|}
\hline Characteristics & Overall & \multicolumn{3}{|c|}{ Liver steatosis } & \multicolumn{3}{|c|}{ Liver fibrosis } \\
\hline Men, \% & 48.20 & 51.72 & 43.16 & $<0.01$ & 56.37 & 46.63 & $<0.01$ \\
\hline Diabetes, \% & 21.95 & 30.51 & 9.72 & $<0.01$ & 39.94 & 18.46 & $<0.01$ \\
\hline Obesity, \% & 42.12 & 57.89 & 19.59 & $<0.01$ & 69.35 & 36.90 & $<0.01$ \\
\hline Education, \% & & & & 0.14 & & & 0.01 \\
\hline$\leq$ High school & 44.26 & 45.18 & 42.93 & & 48.58 & 43.40 & \\
\hline$>$ High school & 55.74 & 54.82 & 57.07 & & 51.42 & 56.60 & \\
\hline Race/Hispanic origin (\%) & & & & $<0.01$ & & & $<0.01$ \\
\hline Mexican American & 13.74 & 16.52 & 9.77 & & 15.86 & 13.33 & \\
\hline Other hispanic & 9.52 & 10.16 & 8.60 & & 9.07 & 9.60 & \\
\hline Smoking status (\%) & & & & $<0.01$ & & & $<0.01$ \\
\hline Current smoker & 17.10 & 15.23 & 19.77 & & 16.86 & 17.14 & \\
\hline Former smoker & 23.53 & 26.68 & 19.04 & & 29.75 & 22.33 & \\
\hline Never smoker & 59.37 & 58.09 & 61.19 & & 53.40 & 60.53 & \\
\hline Physical activity ${ }^{a}$ & & & & $<0.01$ & & & $<0.01$ \\
\hline Yes & 60.24 & 56.73 & 62.70 & & 59.30 & 65.01 & \\
\hline No & 39.76 & 43.27 & 37.30 & & 40.70 & 34.99 & \\
\hline \multicolumn{8}{|l|}{ Dietary intakes of } \\
\hline Vitamin A, RAE ( $\mu \mathrm{g} / \mathrm{d})$ & 605.65 (502.34) & 611.35 (539.20) & $597.51(444.45)$ & 0.40 & $621.65(605.21)$ & 602.61 (479.99) & 0.38 \\
\hline Vitamin B1 (mg/d) & $1.54(0.79)$ & $1.56(0.78)$ & $1.52(0.80)$ & 0.16 & $1.59(0.82)$ & $1.53(0.78)$ & 0.07 \\
\hline Alpha-Carotene ( $\mu \mathrm{g} / \mathrm{d})$ & $396.24(1,154.66)$ & $413.04(1,366.03)$ & $372.25(756.23)$ & 0.28 & $436.00(1,979.85)$ & 388.47 (914.15) & 0.34 \\
\hline Beta-Carotene ( $\mu \mathrm{g} / \mathrm{d})$ & 2,400.22 (4,044.30) & 2,360.12 (4,339.64) & $2,457.50(3,580.95)$ & 0.46 & 2,365.69 (4,969.46) & $2,406.28(3,841.10)$ & 0.82 \\
\hline Beta-Cryptoxanthin $(\mu \mathrm{g} / \mathrm{d})$ & $97.80(251.64)$ & $98.80(256.48)$ & 96.37 (244.62) & 0.77 & $111.74(332.81)$ & 95.09 (232.72) & 0.13 \\
\hline Lutein + zeaxanthin $(\mu \mathrm{g} / \mathrm{d})$ & 1,666.57 (2,936.97) & $1,548.67(2,734.24)$ & 1,834.98 (3,197.73) & $<0.01$ & $1,578.19(2,609.11)$ & 1,683.20 (2,995.67) & 0.41 \\
\hline Lycopene ( $\mu \mathrm{g} / \mathrm{d})$ & 4,498.08 (6,755.69) & $4,482.38(6,645.59)$ & $4,520.50(6,911.93)$ & 0.86 & $4,329.38(6,167.15)$ & $4,531.66(6,862.87)$ & 0.49 \\
\hline Alcohol (g/d) & $2.65(5.74)$ & $2.58(5.72)$ & $2.76(5.76)$ & 0.31 & $2.63(5.65)$ & $2.66(5.77)$ & 0.88 \\
\hline Cholesterol (mg/d) & $298.25(208.27)$ & $304.15(208.15)$ & $289.82(208.22)$ & 0.03 & $319.36(226.25)$ & 294.17 (204.38) & $<0.01$ \\
\hline Serum vitamin C (mg/dL) & $0.80(0.49)$ & $0.85(0.48)$ & $0.97(0.51)$ & $<0.01$ & $0.76(0.48)$ & $0.92(0.49)$ & $<0.01$ \\
\hline
\end{tabular}

Values are means $\pm S D$ s for continuous variables.

a ANOVA test was performed for continuous variables, and Chi-square test was performed for categorical variables.

RAE, retinol activity equivalents; DFE, dietary folate equivalents.

\section{DISCUSSION}

Results from this national population-based survey showed that vitamin $\mathrm{C}, \alpha$-carotene, $\beta$-carotene, lutein + zeaxanthin, choline, and vitamin B12 may be associated with liver steatosis and fibrosis. Dose-response analysis showed that the abovementioned associations were mainly linear, while the evidence of non-linear relationship between $\beta$-carotene and liver steatosis were observed.

Oxidative stress has been reported to be causative in NAFLD initiation and progression $(6,7)$. Day and James proposed that NAFLD requires a double hit: the first hit produces steatosis by a high-fat diet or diabetes, and then the second is a source of oxidative stress capable of initiating significant lipid peroxidation 
TABLE 2 | Associations between vitamins and carotenoids and liver steatosis.

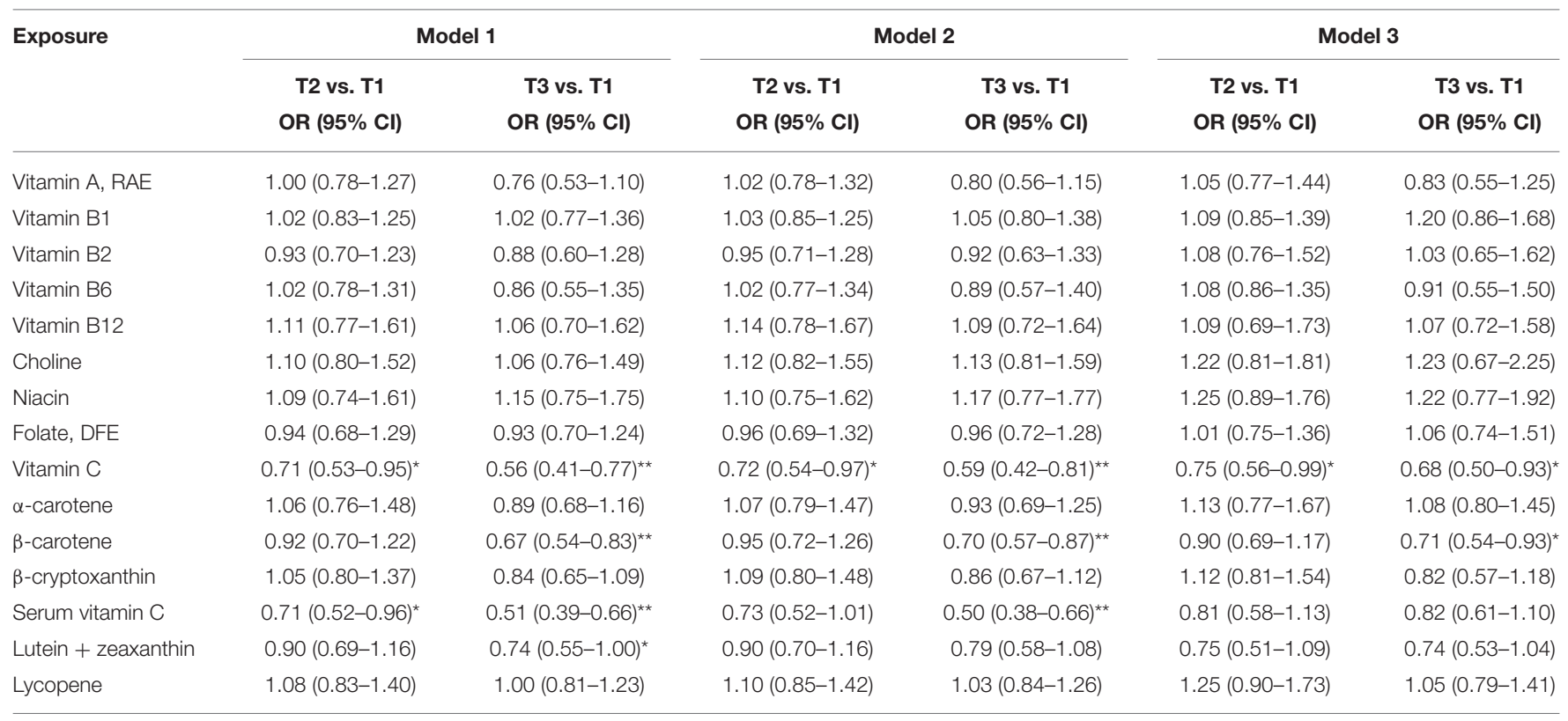

$T 1$, tertile 1; T2, tertile 2; T3, tertile 3.

OR $(95 \% \mathrm{Cl})$, odds ratio (95\% confidence interval).

${ }^{*} P<0.05,{ }^{* *} P<0.01$.

$R A E$, retinol activity equivalents; $D F E$, dietary folate equivalents.

Model 1 was adjusted for age, sex, and race/ethnicity.

Model 2 was adjusted for covariates in model 1, and also education, physical activity, smoking, and alcohol drinking.

Model 3 was adjusted for covariates in model 2, and also body mass index, hypertension, diabetes, and dietary intakes of cholesterol.

TABLE 3 | Associations between vitamins and carotenoids and liver fibrosis.

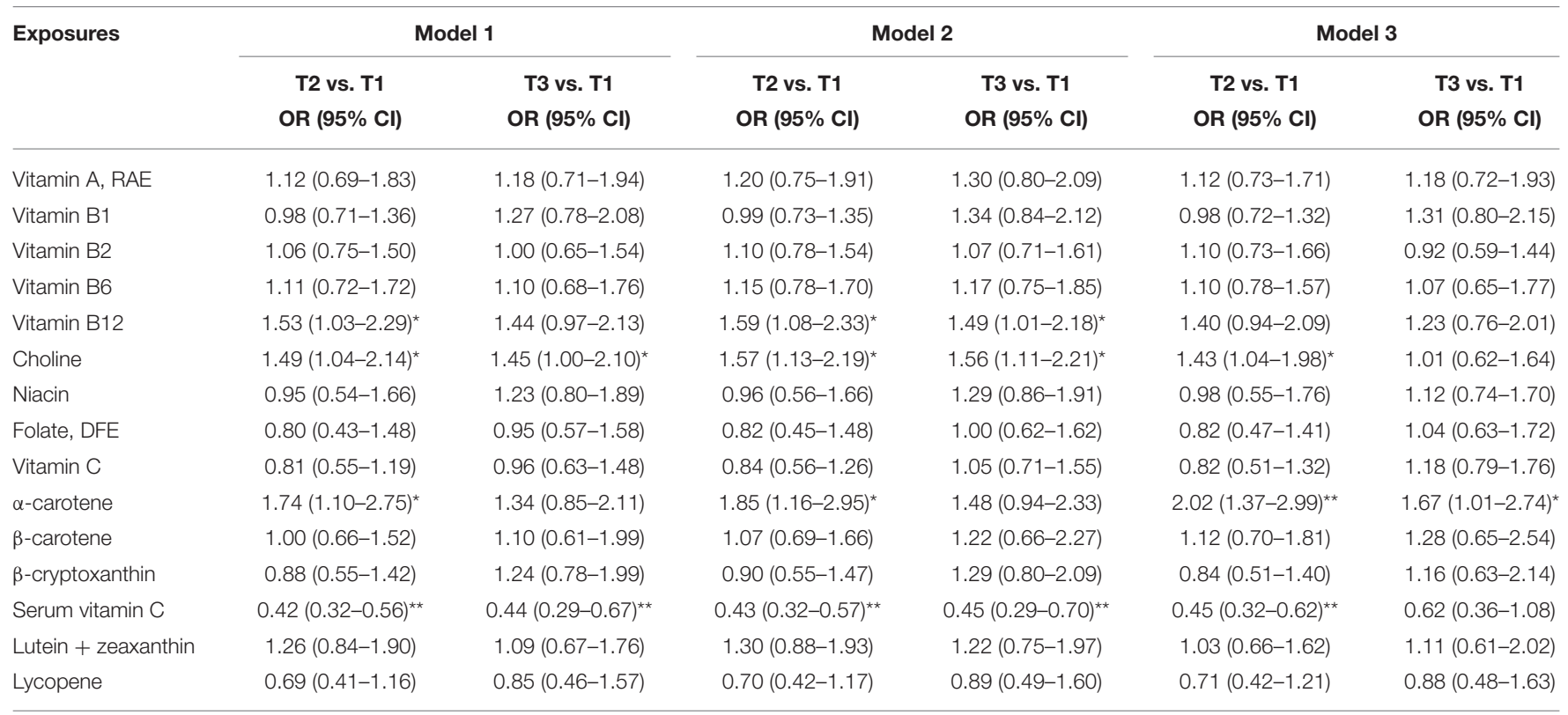

T1, tertile 1; T2, tertile 2; T3, tertile 3.

OR $(95 \% \mathrm{Cl})$, odds ratio ( $95 \%$ confidence interval).

${ }^{*} P<0.05,{ }^{*} P<0.01$.

RAE, retinol activity equivalents; DFE, dietary folate equivalents.

Model 1 was adjusted for age, sex, and race/ethnicity.

Model 2 was adjusted for covariates in model 1, and also education, physical activity, smoking, and alcohol drinking,

Model 3 was adjusted for covariates in model 2, and also body mass index, hypertension, diabetes, and dietary intakes of cholesterol. 
(21). Vitamin C is a powerful antioxidant in human health by scavenging free radicals, which could protect hepatic cells from lipotoxicity-induced cellular oxidative stress $(8,22)$. In addition, vitamin $\mathrm{C}$ is also believed to play a role in circulating and hepatic lipid homeostasis (22). Observational studies on vitamin $C$ and NAFLD are limited. A cross-sectional study in Brazil found a high proportion of inadequate serum vitamin C (27\%) in 72 NAFLD patients (23). Vitamin C intakes were also found lower in NAFLD patients $(N=200)$ than the healthy controls $(N$ $=400$ ) in Iran (24), while no significant difference was found in other case-control studies [120 Jordanian adults (25), 317 Iranians (26), 101 Canadians (27), 52 Indians (28)]. Therefore, previous publications on vitamin C and NAFLD usually included small number of participants. Metabolic comorbidities associated with NAFLD included obesity, type 2 diabetes, hyperlipidemia, hypertension, and metabolic syndrome (1). Vitamin C could improve lipid profile (29), glycemic control and cardiovascular risk factors including hypertension $(30,31)$. A recommended dietary allowance (RDA) of $90 \mathrm{mg} /$ day for adult men and $75 \mathrm{mg} /$ day for adult women is set to provide antioxidant protection (32). Therefore, whether the effect of vitamin $\mathrm{C}$ on liver fibrosis is more evident than that on liver steatosis should be confirmed further.

Vitamin B1 functions as a coenzyme in the metabolism of carbohydrates and branched-chain amino acids, and the RDA for adults is $1.2 \mathrm{mg} /$ day for men and $1.1 \mathrm{mg} /$ day for women (33). Vitamin B12 functions as a coenzyme for a critical methyl transfer reaction, and the $\mathrm{RDA}$ for adults is $2.4 \mu \mathrm{g} /$ day of vitamin B12 (33). The potential mechanisms of vitamin B1 and vitamin B12 in NAFLD remains unclear, and findings from clinical studies are scarce (8). Higher serum levels of vitamin B12 were positively correlated with the severity of steatosis and fibrosis in 614 Brazilian patients (34). Dietary intakes of vitamin B1 and vitamin B12 were also found higher in patients with NAFLD patients than controls in 120 adult Jordanians (25) and 101 Canadians (27). However, the differences of dietary intakes of vitamin B1 and vitamin B12 in 317 Iranians (26) and serum vitamin B12 concentrations in 54 participants in Greece (35) were not significant between with NAFLD patients and controls. In contrast, serum vitamin B12 concentrations were lower in NAFLD patients and controls in 75 Turks (36), and low vitamin B12 levels were significantly associated with a higher fibrosis grade and non-alcoholic steatohepatitis activity in 83 patients in Israel (37). Therefore, previous publications on vitamin B1 and B12 and NAFLD also usually included small number of participants and did not adjust for covariates in univariate analysis. Our results are consistent with those from several publications in which vitamin B1 and B12 levels were higher in NAFLD patients than controls. In addition, higher vitamin B12 levels were associated with a greater risk of total mortality and combined fatal and non-fatal coronary events in prospective cohort studies (38).

Putative mechanisms of other vitamins and carotenoids in NAFLD pathogenesis have been summarized elsewhere (8), and these mechanisms include antioxidant, antifibrotic, immune effects, and lipoprotective effects (8). However, dietary intakes of vitamin A were not associated with liver steatosis and liver fibrosis in this analysis, and the inverse association between $\beta$-carotene and liver steatosis was not significant after further adjustment for metabolic comorbidities associated with NAFLD including obesity, diabetes, and hypertension. Previous studies on vitamin $\mathrm{A}(23-27,39)$ and $\beta$-carotene $(23,26)$ are also limited and not consistent. As shown above, previous studies usually included small number of participants and did not adjust for other covariates.

To our knowledge, this is the first national populationbased survey to explore the associations between these vitamins and carotenoids and liver fibrosis and steatosis. We also explored the potential non-linear dose-response relationships and considered a number of covariates. However, there are also limitations within this study. First, this is a cross-sectional study and we cannot determine the causality, which should be confirmed by prospective studies. In addition, patients may change their dietary habits after diagnosis of NAFLD. However, studies on these vitamins and carotenoids are limited, and there are no clinical guidelines to date recommending or limiting these vitamins and carotenoids intake for NAFLD prevention. Second, misclassification of these vitamins and carotenoids consumption could be of concern; however, nondifferential misclassification at baseline should have weakened the association. Third, although we considered a number of covariates, the extent to which they were adjusted and residual confounding by other unmeasured factors are also of concern in observational studies. However, the findings were similar between model 1 and model 2, although several associations were not significant after further adjustment for obesity, diabetes, and hypertension. Finally, a number of participants were excluded from this analysis. In a recent study using data from the 20172018 NHANES cycle, the prevalence of non-alcoholic fatty liver disease (controlled attenuation parameter scores of $\geq 248 \mathrm{~dB} / \mathrm{m}$ ) was $56.7 \%$, and the prevalence of significant liver fibrosis $(\geq 7.9$ $\mathrm{kPa}$ ) was $14.5 \%$ (14), which are comparable to the findings in this study.

In summary, results from this national population-based survey showed that vitamin $\mathrm{C}, \alpha$-carotene, $\beta$-carotene, lutein + zeaxanthin, choline, and vitamin B12 may be associated with liver steatosis and fibrosis. The above-mentioned associations were mainly linear, while the relationship between $\beta$-carotene and liver steatosis might be non-linear. These findings need to be confirmed by prospective studies.

\section{DATA AVAILABILITY STATEMENT}

The datasets generated during and/or analyzed during the current study are available in the NHANES: https://www.cdc.gov/ nchs/nhanes/.

\section{ETHICS STATEMENT}

NHANES was approved by the National Center for Health Statistics Research Ethics Review Board. Ethical approval for this study is deemed exempt because this study uses publically 
available secondary data. The patients/participants provided their written informed consent to participate in this study.

\section{AUTHOR CONTRIBUTIONS}

XL and JS: conceptualization. XL: methodology and resources. $\mathrm{XL}$ and HS: formal analysis. XL, HS, and MC: writing. JS: supervision. All authors contributed to the article and approved the submitted version.

\section{REFERENCES}

1. Younossi ZM, Koenig AB, Abdelatif D, Fazel Y, Henry L, Wymer M. Global epidemiology of nonalcoholic fatty liver disease-Meta-analytic assessment of prevalence, incidence, and outcomes. Hepatology. (2016) 64:73-84. doi: 10.1002/hep.28431

2. Abdelmalek MF. Nonalcoholic fatty liver disease: another leap forward. Nat Rev Gastroenterol Hepatol. (2021) 18:85-6. doi: 10.1038/s41575-020-00406-0

3. Sanyal AJ. Past, present and future perspectives in nonalcoholic fatty liver disease. Nat Rev Gastroenterol Hepatol. (2019) 16:377-86. doi: 10.1038/s41575-019-0144-8

4. Younossi ZM, Corey KE, Lim JK. AGA clinical practice update on lifestyle modification using diet and exercise to achieve weight loss in the management of nonalcoholic fatty liver disease: expert review. Gastroenterology. (2021) 160:912-8. doi: 10.1053/j.gastro.2020.11.051

5. Parra-Vargas M, Rodriguez-Echevarria R, Jimenez-Chillaron JC. Nutritional approaches for the management of nonalcoholic fatty liver disease: an evidence-based review. Nutrients. (2020) 12:3860. doi: 10.3390/nu12123860

6. Rives C, Fougerat A, Ellero-Simatos S, Loiseau N, Guillou H, Gamet-Payrastre L, et al. Oxidative stress in NAFLD: role of nutrients and food contaminants. Biomolecules. (2020) 10:1702. doi: 10.3390/biom10121702

7. Chen G, Ni Y, Nagata N, Xu L, Ota T. Micronutrient antioxidants and nonalcoholic fatty liver disease. Int J Mol Sci. (2016) 17:1379. doi: 10.3390/ijms17091379

8. Pickett-Blakely O, Young K, Carr RM. Micronutrients in nonalcoholic fatty liver disease pathogenesis. Cell Mol Gastroenterol Hepatol. (2018) 6:451-62. doi: 10.1016/j.jcmgh.2018.07.004

9. Barchetta I, Cimini FA, Cavallo MG. Vitamin D and metabolic dysfunctionassociated fatty liver disease (MAFLD): an update. Nutrients. (2020) 12:3302. doi: $10.3390 /$ nu 12113302

10. Abdel-Maboud M, Menshawy A, Menshawy E, Emara A, Alshandidy M, Eid $\mathrm{M}$. The efficacy of vitamin $\mathrm{E}$ in reducing non-alcoholic fatty liver disease: a systematic review, meta-analysis, and meta-regression. Therap Adv Gastroenterol. (2020) 13:1756284820974917. doi: 10.1177/1756284820974917

11. Castera L, Friedrich-Rust M, Loomba R. Noninvasive assessment of liver disease in patients with nonalcoholic fatty liver disease. Gastroenterology. (2019) 156:1264-81.e4. doi: 10.1053/j.gastro.2018.12.036

12. Pu K, Wang Y, Bai S, Wei H, Zhou Y, Fan J, et al. Diagnostic accuracy of controlled attenuation parameter (CAP) as a non-invasive test for steatosis in suspected non-alcoholic fatty liver disease: a systematic review and metaanalysis. BMC Gastroenterol. (2019) 19:51. doi: 10.1186/s12876-019-0961-9

13. Kim D, Konyn P, Cholankeril G, Ahmed A. Physical activity is associated with nonalcoholic fatty liver disease and significant fibrosis measured by fibroscan. Clin Gastroenterol Hepatol. (2021). doi: 10.1016/j.cgh.2021.06.029. [Epub ahead of print].

14. Zhang X, Heredia NI, Balakrishnan M, Thrift AP. Prevalence and factors associated with NAFLD detected by vibration controlled transient elastography among US adults: results from NHANES 2017-2018. PLoS ONE. (2021) 16:e0252164. doi: 10.1371/journal.pone.0252164

15. Liver Ultrasound Transient Elastography Procedures Manual. Centers for Disease Control and Prevention (2018). Available online at: https://wwwn. cdc.gov/nchs/data/nhanes/2017-2018/manuals/2018_Liver_Ultrasound_ Elastography_Procedures_Manual.pdf

16. Eddowes PJ, Sasso M, Allison M, Tsochatzis E, Anstee QM, Sheridan D, et al. Accuracy of FibroScan controlled attenuation parameter and

\section{ACKNOWLEDGMENTS}

We thank all participants in the NHANES.

\section{SUPPLEMENTARY MATERIAL}

The Supplementary Material for this article can be found online at: https://www.frontiersin.org/articles/10.3389/fnut.2021. 760985/full\#supplementary-material

liver stiffness measurement in assessing steatosis and fibrosis in patients with nonalcoholic fatty liver disease. Gastroenterology. (2019) 156:1717-30. doi: 10.1053/j.gastro.2019.01.042

17. Siddiqui MS, Vuppalanchi R, Van Natta ML, Hallinan E, Kowdley KV, Abdelmalek M, et al. Vibration-Controlled transient elastography to assess fibrosis and steatosis in patients with nonalcoholic fatty liver disease. Clin Gastroenterol Hepatol. (2019) 17:156-63.e2. doi: 10.1016/j.cgh.2018.04.043

18. Menke A, Casagrande S, Geiss L, Cowie CC. Prevalence of and trends in diabetes among adults in the United States, 1988-2012. JAMA. (2015) 314:1021-9. doi: 10.1001/jama.2015.10029

19. Whelton PK, Carey RM, Aronow WS, Casey DE Jr, Collins KJ, Dennison Himmelfarb C, et al. 2017 ACC/AHA/AAPA/ $\mathrm{ABC} / \mathrm{ACPM} / \mathrm{AGS} / \mathrm{APhA} / \mathrm{ASH} / \mathrm{ASPC} / \mathrm{NMA} / \mathrm{PCNA}$ guideline for the prevention, detection, evaluation, and management of high blood pressure in adults: executive summary: a report of the American college of cardiology/American heart association task force on clinical practice guidelines. J Am Coll Cardiol. (2018) 71:2199-269. doi: 10.1161/HYP.0000000000000075

20. Desquilbet L, Mariotti F. Dose-response analyses using restricted cubic spline functions in public health research. Stat Med. (2010) 29:1037-57. doi: $10.1002 / \operatorname{sim} .3841$

21. Day CP, James OF. Steatohepatitis: a tale of two "hits"? Gastroenterology. (1998) 114:842-5. doi: 10.1016/S0016-5085(98)70599-2

22. Raza S, Tewari A, Rajak S, Sinha RA. Vitamins and non-alcoholic fatty liver disease: a molecular insight (small star, filled). Liver Res. (2021) 5:62-71. doi: 10.1016/j.livres.2021.03.004

23. Coelho JM, Cansancao K, Perez RM, Leite NC, Padilha P, Ramalho A, et al. Association between serum and dietary antioxidant micronutrients and advanced liver fibrosis in non-alcoholic fatty liver disease: an observational study. PeerJ. (2020) 8:e9838. doi: 10.7717/peerj.9838

24. Lotfi A, Saneei P, Hekmatdost A, Salehisahlabadi A, Shiranian A, Ghiasvand R. The relationship between dietary antioxidant intake and physical activity rate with nonalcoholic fatty liver disease (NAFLD): a case - control study. Clin Nutr ESPEN. (2019) 34:45-9. doi: 10.1016/j.clnesp.2019.09.004

25. Tayyem RF, Al-Dayyat HM, Rayyan YM. Relationship between lifestyle factors and nutritional status and non-alcoholic fatty liver disease among a group of adult Jordanians. Arab J Gastroenterol. (2019) 20:44-9. doi: 10.1016/j.ajg.2019.01.008

26. Zolfaghari H, Askari G, Siassi F, Feizi A, Sotoudeh G. Intake of nutrients, fiber, and sugar in patients with nonalcoholic fatty liver disease in comparison to healthy individuals. Int J Prev Med. (2016) 7:98. doi: $10.4103 / 2008-7802.188083$

27. Da Silva HE, Arendt BM, Noureldin SA, Therapondos G, Guindi M, Allard JP. A cross-sectional study assessing dietary intake and physical activity in Canadian patients with nonalcoholic fatty liver disease vs healthy controls. J Acad Nutr Diet. (2014) 114:1181-94. doi: 10.1016/j.jand.2014.01.009

28. Madan K, Bhardwaj P, Thareja S, Gupta SD, Saraya A. Oxidant stress and antioxidant status among patients with nonalcoholic fatty liver disease (NAFLD). J Clin Gastroenterol. (2006) 40:930-5. doi: 10.1097/01.mcg.0000212608.59090.08

29. Namkhah Z, Ashtary-Larky D, Naeini F, Clark CCT, Asbaghi O. Does vitamin $\mathrm{C}$ supplementation exert profitable effects on serum lipid profile in patients with type 2 diabetes? A systematic review and dose-response meta-analysis. Pharmacol Res. (2021) 169:105665. doi: 10.1016/j.phrs.2021.1 05665 
30. Mason SA, Keske MA, Wadley GD. Effects of vitamin C supplementation on glycemic control and cardiovascular risk factors in people with type 2 diabetes: a GRADE-assessed systematic review and meta-analysis of randomized controlled trials. Diabetes Care. (2021) 44:618-630. doi: 10.2337/ dc20-1893

31. Ran L, Zhao W, Tan X, Wang H, Mizuno K, Takagi K, et al. Association between serum vitamin $\mathrm{C}$ and the blood pressure: a systematic review and meta-analysis of observational studies. Cardiovasc Ther. (2020) 2020:4940673. doi: $10.1155 / 2020 / 4940673$

32. Institute of Medicine (US) Panel on Dietary Antioxidants and Related Compounds. Dietary Reference Intakes for Vitamin C, Vitamin E, Selenium, and Carotenoids. Washington, DC: Institute of Medicine (US) Panel on Dietary Antioxidants and Related Compounds (2000).

33. Thiamin R. Dietary Reference Intakes for Thiamin, Riboflavin, Niacin, Vitamin B6, Folate, Vitamin B12, Pantothenic Acid, Biotin, and Choline. Washington, DC: National Academies Press (1998). doi: 10.17226/6015

34. Bertol FS, Araujo B, Jorge BB, Rinaldi N, De Carli LA, Tovo CV. Role of micronutrients in staging of nonalcoholic fatty liver disease: a retrospective cross-sectional study. World J Gastrointest Surg. (2020) 12:26976. doi: 10.4240/wjgs.v12.i6.269

35. Polyzos SA, Kountouras J, Patsiaoura K, Katsiki E, Zafeiriadou E, Zavos C, et al. Serum vitamin B12 and folate levels in patients with nonalcoholic fatty liver disease. Int J Food Sci Nutr. (2012) 63:659-66. doi: 10.3109/09637486.2011.649249

36. Koplay M, Gulcan E, Ozkan F. Association between serum vitamin B12 levels and the degree of steatosis in patients with nonalcoholic fatty liver disease. J Investig Med. (2011) 59:1137-40. doi: 10.2310/JIM.0b013e3182 $2 \mathrm{a} 29 \mathrm{f5}$
37. Mahamid M, Mahroum N, Bragazzi NL, Shalaata K, Yavne Y, Adawi M, et al. Folate and B12 levels correlate with histological severity in NASH patients. Nutrients. (2018) 10:440. doi: 10.3390/nu10040440

38. Rafnsson SB, Saravanan P, Bhopal RS, Yajnik CS. Is a low blood level of vitamin B12 a cardiovascular and diabetes risk factor? A systematic review of cohort studies. Eur J Nutr. (2011) 50:97-106. doi: 10.1007/s00394-010-0119-6

39. Liu Y, Chen H, Wang J, Zhou W, Sun R, Xia M. Association of serum retinoic acid with hepatic steatosis and liver injury in nonalcoholic fatty liver disease. Am J Clin Nutr. (2015) 102:130-7. doi: 10.3945/ajcn.114.105155

Conflict of Interest: The authors declare that the research was conducted in the absence of any commercial or financial relationships that could be construed as a potential conflict of interest.

Publisher's Note: All claims expressed in this article are solely those of the authors and do not necessarily represent those of their affiliated organizations, or those of the publisher, the editors and the reviewers. Any product that may be evaluated in this article, or claim that may be made by its manufacturer, is not guaranteed or endorsed by the publisher.

Copyright (c) 2021 Liu, Shen, Chen and Shao. This is an open-access article distributed under the terms of the Creative Commons Attribution License (CC BY). The use, distribution or reproduction in other forums is permitted, provided the original author(s) and the copyright owner(s) are credited and that the original publication in this journal is cited, in accordance with accepted academic practice. No use, distribution or reproduction is permitted which does not comply with these terms. 